\title{
How situational activation of values evokes positive and negative feelings: Theory and experimental findings
}

\author{
William Montgomery ${ }^{1}\left[\right.$ D $\cdot$ Henry Montgomery ${ }^{2,3} \cdot$ Tommy Gärling $^{4}$
}

Published online: 20 January 2020

(c) The Author(s) 2020

\begin{abstract}
We propose a theory of how situational activation of values evokes positive and negative feelings. In conjunction we present a re-conceptualization of Schwartz' et al. (J Personal Soc Psychol 103:663-688, 2012. https://doi.org/10.1037/a029393) value set including additional values. In our new value set, we posit contrastive values having opposite values and central values having no opposite values. As a consequence, balanced access to salient opposite contrastive values and maximal access to central values evoke the strongest positive and weakest negative feelings. Study 1 shows, as hypothesized, that contrastive values form a circumplex structure with central values located inside its periphery. Study 2 supports theoretically derived hypotheses of how positive and negative feelings are evoked by different degrees of access to values, salience of opposite values, and centrality of values.
\end{abstract}

Keywords Values $\cdot$ Circumplex structure $\cdot$ Feeling $\cdot$ Salience $\cdot$ Decisions

Values and emotions are central constructs in contemporary psychology (Feldman Barrett et al. 2016; Maio 2016) that typically are studied isolated from each other, although both have in common that they motivate human behavior (Baumeister 2007; Tamir et al. 2016; Verplanken and Holland 2002). Values are commonly defined as desirable goals that motivate behavior but transcend specific behaviors and situations (Schwartz 2010). Yet, some values (e.g., Security and Courage in our revised value structure presented below) may be in conflict with each other such that if one is accessed, the other cannot be accessed. We define a person's

William Montgomery

william.montgomery@hig.se

Henry Montgomery

hmy@psychology.su.se

Tommy Gärling

tommy.garling@psy.gu.se

1 Academy of Health and Occupational Studies, University of Gävle, 80176 Gävle, Sweden

2 Department of Psychology, Stockholm University, 16091 Stockholm, Sweden

3 Uppsala University, Uppsala, Sweden

4 Department of Psychology, University of Gothenburg, Box 500, 40530 Göteborg, Sweden access to a value as depending on an appraisal of the degree of congruence between the value and the person's actual situation. For example, if a person experiences that s/he has $50 \%$ access to courage this means that the person appraises that there is $50 \%$ congruence between the value courageous and the person's actual level of courage. This example illustrates that people perceive conflicts between the possibility of getting simultaneous access to pairs of contrastive values such as security and courage. The point of departure for the present paper is the assumption that conflicts between values evoke emotions or feelings, at the same time as the structure of values depends on how access to values is related to emotions or feelings.

Verplanken and Holland (2002) and Maio et al. (2009) have shown that situations activate values. When values are activated, they typically also evoke emotions (Beck et al. 1979; Johnson-Laird and Oatley 1992; Mesquita and Frijda 2011). Although emotions have different properties including experiential, behavioral and physiological responses (Russell and Feldman Barrett 1999), in this paper we analyze the experiential aspect of emotion, which we denote feeling. Specifically, our focus is on conscious positive versus negative feelings varying in the valence dimension of the affect grid proposed by Russell (1980), (2003) and Yik et al. (2011). For instance, in a situation that activates the value of courage, a person would feel excited (positive feeling) if 
he or she prefers courage to security but would feel bored (negative feeling) if the situation instead activates security. The reverse would be the case if the person prefers security to courage. We thus propose that positive or negative feelings are evoked by appraisals of a situation as being congruent with specific values. This proposal may be viewed as a special case of appraisal theories of emotions, which assume that emotions result from appraisals of a given situation as being congruent with a number of criteria (Moors et al. 2013; Scherer 1999).

A few empirical studies have investigated how inter-individual variation in value priorities are related to dispositions to activate specific emotions (Koopman-Holm and Matsumoto 2011; Nelissen et al. 2007; Schwartz 2012; Silfver et al. 2008). For example, Nelissen et al. (2007) reported correlations across individuals between priorities of values in Schwartz' value set and incidence of specific emotions. By contrast, our aim is to study the link between values and feelings at the intra-individual level. Specifically, our aim is to investigate how activation of specific values evokes positive and negative feelings.

\section{Value structure}

\section{Schwartz' theory of value structure}

In the last 30 years the research by Schwartz (e.g., 1992) has had an immense impact on the understanding of human values. Schwartz hypothesized that 10 basic human values form a two-dimensional circumplex structure (see Fig. 1). The values include Power, Achievement, Hedonism, Stimulation,

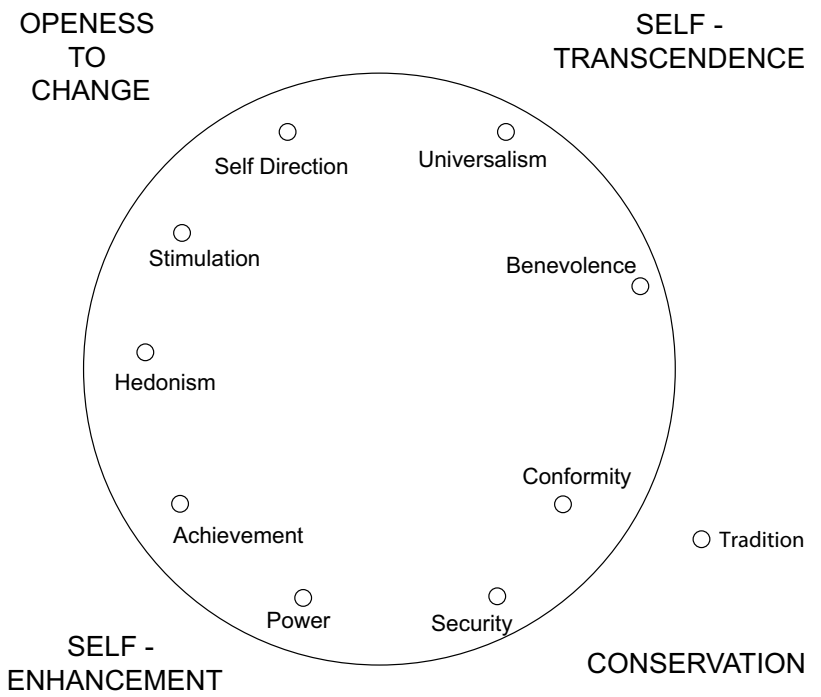

Fig. 1 Theoretical model of relations among 10 motivational types of values (after Schwartz and Boehnke 1992)
Self-Direction, Universalism, Benevolence, Conformity, Tradition, and Security. The 10 values are mapped on two bipolar dimensions, defined in terms of conceptually contrasting motivations: Self Enhancing-Self-Transcendence and Openness to Change-Conservation.

Schwartz (2007) found support for the hypothesized value structure by means of multidimensional scaling (MDS) of data from 195 samples of teachers, students, and lay people in 66 countries (see also Schwartz and Bardi 2001). Figure 1 shows that the structure is not strictly circular since tradition and conformity are overlapping each other. Recently, Schwartz' model was cross-validated by neuroimaging data (Leszkowicz et al. 2017; Zacharopoulos et al. 2017). Schwartz et al. (2012) refined the theory by partitioning some of the 10 value into more specific values and by adding two new values interspersed between the others (Face between Security and Power, and Humility between Conformity and Benevolence), thus resulting in a total of 19 values.

\section{Contrastive and central values}

We propose an alternative set of values which we argue is a more adequate basis for understanding the relationship between values and feelings at an individual level. The value structure by Schwartz (1992) had nine positions in the circumplex structure (Tradition were outside and not next to Conformity) and the value structure by Schwartz (2012) had 19 positions in the circumplex. These are uneven numbers of positions in the circumplex and therefore his value set cannot be divided into pairs which are opposite each other in the structure. As shown below, we have therefore re-conceptualized Schwartz et al.'s (2012) set of values and added some values that are partly different from Schwartz' values. At the same time, we share Schwartz' idea that some basic human values may be organized in a circumplex structure, although, as will be discussed below, we have also added values that are located inside the circumplex.

In Table 1 we present our values including eight contrastive values, each of which has another value as a counterpart located opposite in a circumplex. The four pairs of contrastive values are Security-Courage, Adaptation-Own Will, Management by Yourself-Get Help, and Prioritize Others-Prioritize Yourself.

We assume that besides the contrastive values, there are additional values that are located inside the circumplex. We interpret our set of values as a two-level value hierarchy. The contrastive values located on the periphery of the circumplex represent the lower level. Access to these values are instrumental for getting access to higher level values that are located inside the circumplex and called central values. For example, Management by Yourself could be seen as a means to getting access to the higher order values Respect 
Table 1 Contrastive value pairs and central values

\begin{tabular}{|c|c|c|}
\hline Value type & Value & Opposite value \\
\hline Contrastive & Management by yourself & Get help \\
\hline Contrastive & $\begin{array}{l}\text { Getting through your own } \\
\text { will }\end{array}$ & Adaptation to others wishes \\
\hline Contrastive & Prioritize oneself & Prioritize others \\
\hline Contrastive & Security & Courage \\
\hline Central & $\begin{array}{l}\text { Having deeper understand- } \\
\text { ing about the world }\end{array}$ & - \\
\hline Central & Being healthy & - \\
\hline Central & Making achievement & - \\
\hline Central & To be respected & - \\
\hline Central & Experience enjoyment & - \\
\hline Central & Experience happiness & - \\
\hline Central & $\begin{array}{l}\text { Experience meaningful- } \\
\text { ness }\end{array}$ & - \\
\hline Central & Experience love & - \\
\hline
\end{tabular}

and Achievement. There are eight central values (Happiness, Love, Meaningfulness, Respect, Understanding, Achievement, Health, and Enjoyment). The central values do not have opposite values. Therefore, increased access to them leads to more positive and less negative feelings.

Table 2 shows that Schwartz et al.'s (2012) values coincide with or are close to nine of our values (Security, Courage, Adaptation, Own Will, Prioritize Others, Being Respected, Enjoyment, Achievement, and Management by Yourself). Enjoyment is close to Hedonism, Being Respected is close to Face, and Own Will is close to Power. Courage is more altruistically biased than Stimulation, which implies partially different relations to other values, but both values are different kinds of risk taking which may lead to excitement or fear. Being high in access to Management by Yourself implies freedom to cultivating own ideas and abilities as well as determining own actions. Therefore, Management by Yourself covers Self Direction as defined by Schwartz. Schwartz' values Humility, Benevolence, and Universalism

Table 2 Description of Schwartz' (2012) value structure compared to current values

\begin{tabular}{ll}
\hline Schwartz Comparisons with current values & Cons
\end{tabular}

Self-direction-thought Freedom to cultivate one's own ideas and abilities

The possibility to manage things by yourself when it is about cultivating your own ideas and abilities

Self-direction-action Freedom to determine one's own actions

The possibility to manage things by yourself when it is about determining your own actions

Stimulation Excitement, novelty, and change

Excitement: positive emotion of making something courageous Novelty: being courageous to make something new Change: being courageous to make a change

Hedonism Pleasure and sensuous gratification

Enjoyment out of pleasure and sensuous gratification

Achievement Success according to social standards

Power-dominance Power through exercising control over people

Power-resources Power through control of material and social resources

Success according to social standards through achievement

To get your own will through when it is about control over people

To get your own will through when it is about control of material and social resources

Face Security and power through maintaining one's public image and avoiding humiliation

Being respected through maintaining one's public image and avoiding humiliation

Security in one's immediate environment

Security in the wider society

Adaptation to cultural, family, or religious traditions' wishes

Tradition Maintaining and preserving cultural, family, or religious traditions

Conformity-rules Compliance with rules, laws, and formal obligations

Conformity-interpersonal Avoidance of upsetting or harming other people

Humility Recognizing one's insignificance in the larger scheme of things

Benevolence-dependability Being a reliable and trustworthy member of the in-group

Benevolence-caring Devotion to the welfare of in-group members

Universalism-concern Commitment to equality, justice, and protection for all people

Universalism-nature Preservation of the natural environment

Universalism-tolerance Acceptance and understanding of those who are different from oneself

Adaptation to rules, laws, and formal obligations

Adaptation to other people by not upsetting or harming them

Prioritize others by recognizing one's insignificance in the larger scheme of things

Prioritize others through being honest and reliable

Prioritize others through devotion to the welfare of in-group members

Prioritize others in the world through commitment to equality, justice, and protection for all people

Prioritize others in the nature through preserving it

Prioritize others by accepting and understanding those who are different from you 
are different kinds of Prioritize Others, where Humility is more focused on mental aspects, Universalism more focused on people at large, and Benevolence more focused on ingroups. Schwartz' values Tradition and Conformity are different kinds of Adaptation, where Conformity is focused on adaptation to rules while Tradition is focused on adaptation to traditions.

Seven additional values (Get Help, Prioritize Yourself, Health, Love, Meaningfulness, Deeper Understanding, and Happiness) do not have clear counterparts in Schwartz et al.'s (2012) value structure. Health was discarded by Schwartz et al. (2012) as a possible Security-Personal value. In any case it lacked a stable meaning across cultures. Health is close to Security, but also to Enjoyment and Happiness, which means that it cannot be seen as a pure instance of Security. Prioritize Yourself is close to Hedonism (Enjoyment), Achievement, and Power (Own will), but access to these values does not necessarily mean prioritizing yourself before prioritizing others, which we assume is the case when the value Prioritize Yourself is focused. Universalism-Concern includes one item which partially explains Deeper Understanding (Understanding for People), but there are other aspects of Deeper Understanding which are not related to understanding people (e.g., understanding of theoretical physics). The remaining values (Get Help, Love, Meaningfulness, and Happiness) are not closely related to any of the values in Schwartz et al. (2012). Finally, it should be noted that Achievement and Enjoyment in our value structure are "semi-central" values, that is positioned towards the center of the circumplex, whereas they correspond to values in the periphery of Schwartz' value structure (Enjoyment assumed to correspond to hedonism).

We conclude that Schwartz et al.'s (2012) values do not include all the values that we propose in our modified value set, at least not at the individual level. However, Health, Love, Deeper Understanding, and Meaningfulness were included in a value structure proposed earlier by Schwartz (1992) (including Healthy, Mature Love, True Friendship, Wisdom, and Meaning in Life), but were removed since found to have different meanings across cultures (Schwartz 2001), and hence, did not consistently fit empirically into any of Schwartz' (1992) values. In our value structure, the values are central. Items that explain certain aspects of Get Help and Happiness (Reciprocation and Inner Harmony) were included in Schwartz' (1992) value structure, but these items are not completely consistent with our values. One may Get Help without giving a favor first and one can at least sometimes experience Happiness without Inner harmony. Also, these items were later removed in Schwartz and Bardi (2001) for having different meanings across cultures.

Since some of the values in our value set have different meanings across cultures (Schwartz 1992; Schwartz and Bardi 2001) we cannot claim that our value structure is consistent across cultures.

\section{Study 1}

The aim of Study 1 was to investigate the validity of our proposed value set. We tested the following two hypotheses:

Hypothesis 1 . The contrastive values form a circumplex value structure with central values inside the periphery.

Hypothesis 2. The contrastive values can be divided into four pairs where the first value in a pair is at the opposite side of the circumplex structure than the second value in a pair (Security-Courage, Adaptation-Own Will, Management by Yourself-Get Help, and Prioritize Others-Prioritize Yourself).

\section{Method}

Two samples were recruited. In Sample 1, 143 undergraduates at the Department of Psychology, Stockholm University, participated in compensation for course credit. Forty-eight were men and 95 were women. Their mean age was 29.30 years and varied between 19 and 72 years $(S D=7.92)$. They were invited through email to answer an online questionnaire in Swedish.

The values were translated into English (as in Table 1) and included in another online questionnaire answered by the participants in Sample 2 consisting of 100 US residents recruited by the internet site Amazon Mechanical Turk. Fifty-one were women and 49 were men. Their mean age was 35.60 years and varied between 20 and 72 years $(S D=9.92)$. They were compensated with US\$ 1 .

In the questionnaires, the participants were first asked about their age and gender. In order to familiarize the participants with the values, they were then asked to use 9-point scales to rate their access to the 8 contrastive and 8 central values. Thereafter, descriptions were presented of fictitious persons who had high access to one of the values asking the participants to rate on 9-point scales ranging from "a very small extent" to "a very large extent" how much access the person has to another value. All pairs of values were presented in individually randomized orders. The instructions read (translated from Swedish): "You will read descriptions of different persons who have high access to one of the values. Rate on the scales beneath how much access you think the person has to the other value presented at the same time."

\section{Results}

The complete matrix of mean compatibility ratings for each pair of values was submitted to MDS (implying that compatibility ratings are inversely related to distances between the values in the MDS) using the PROXSCAL procedure in SPSS. The results are plotted in Fig. 2. Normalized raw stress was 0.04 in the Swedish sample and 0.03 in the US sample, which are close to excellent fits (Cohen 1988). The structures are remarkably similar between the two samples as well as being 

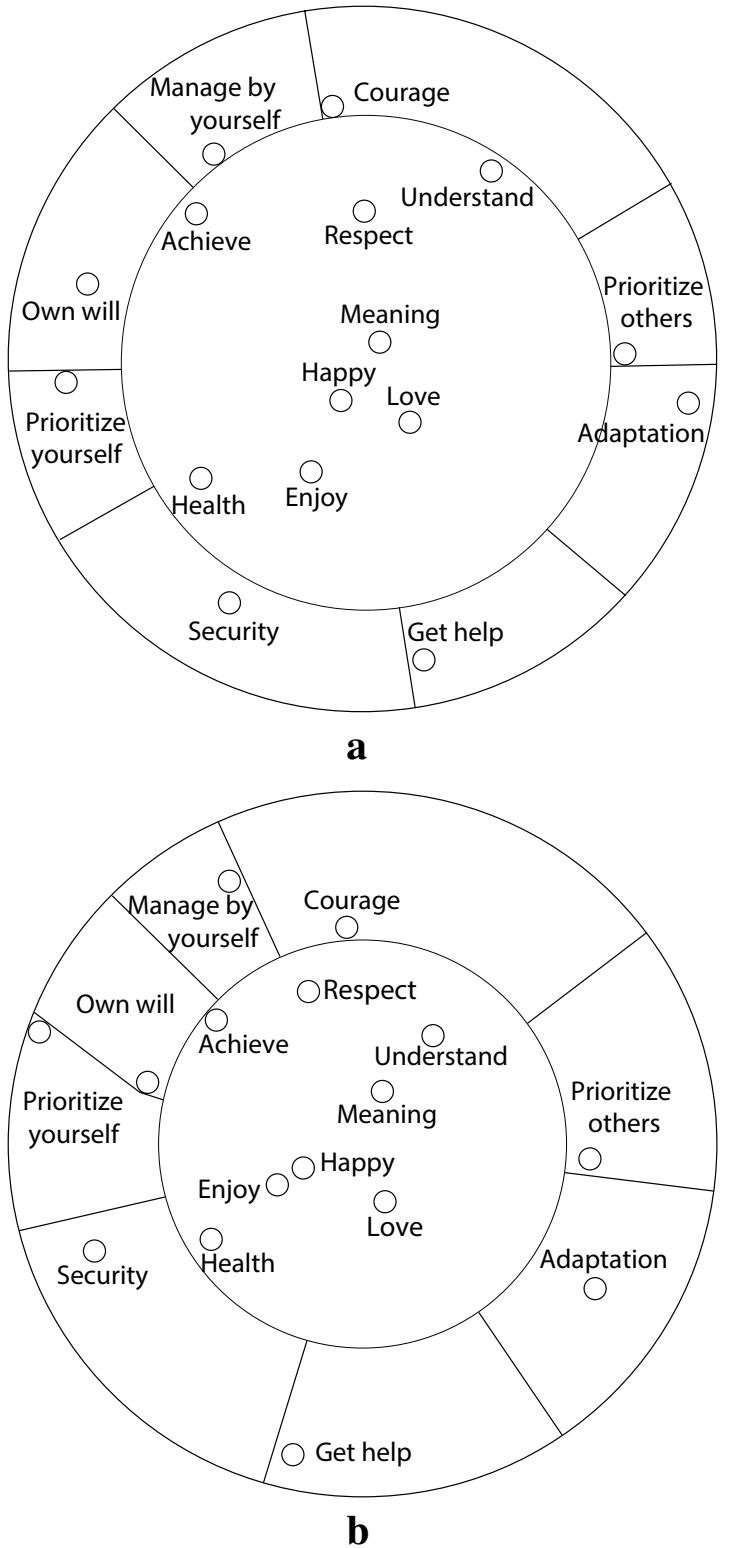

Fig. 2 Multidimensional scaling of ratings of compatibility between contrastive and central values. a Swedish sample. b US sample

closely in line with Hypotheses 1 and 2, except for Prioritize Yourself and Own Will in the US sample which are located at approximately the same angle.

\section{Theory}

In the following we develop a theory of how positive and negative feelings are related to the central and contrastive values. This is followed by presenting Study 2 that tests hypotheses derived from the theory's propositions.

We propose first that the degree of positive or negative feelings evoked by an activated value increases with the degree of access the individual has to the value depending on his or her appraisal of its congruence with the situation. For contrastive values, access is stipulated to vary from $0 \%$ access to one value and simultaneous $100 \%$ access to the opposite value. Thus, the percentages of access to a contrastive value and its opposite are complementary, for instance $25 \%$ and $75 \%, 50 \%$ and $50 \%$, or $75 \%$ and $25 \%$, such that the sum is always $100 \%$. For central values, a positive feeling increases and a negative feeling decreases with the degree of access to the value. For example, an individual would feel most positive and least negative when having $100 \%$ access to Happiness and feel less positive and somewhat more negative if having $70 \%$ access to Happiness and thus lack $30 \%$ access to Happiness.

In order to further specify how contrastive values evoke feelings, we suggest that Coombs' (1950) ideal-point model is applicable. According to this model, an individual's preference for an object is proportional to the distance between the object and an ideal point in a unidimensional or multidimensional space (in our case the unidimensional continuum of access to the value). We accordingly propose that positive feelings decrease and negative feelings increase proportional to the distance between the degree of access to the value and the ideal point of access. Formally, we thus propose that

$\mathrm{F}_{\mathrm{p}}=\mathrm{a}_{\mathrm{p}}+\mathrm{b}_{\mathrm{p}}\left(1-\left|\mathrm{A}-\mathrm{I}_{\mathrm{p}}\right|\right)$,

$\mathrm{F}_{\mathrm{n}}=\mathrm{a}_{\mathrm{n}}+\mathrm{b}_{\mathrm{n}}\left|\mathrm{A}-\mathrm{I}_{-}\right|$,

where $F_{p}$ denotes positive feelings, $F_{n}$ denotes negative feelings, $A$ is the access level of a given value (ranging from 0 minimal access to 1 maximal access), $I_{p}$ and $I_{n}$ are the access levels that correspond to the ideal point for positive and negative feelings, respectively. $a_{p}$, and $a_{n}$ are the intercepts, $b_{p}$, and $b_{n}$ the slopes of the linear relationships.

To illustrate, $40 \%$ access to Security and $60 \%$ access to Courage may be an individual's ideal point for the positive feelings of calmness and excitement that are associated with these levels of access and, hence, the maximum degree of positive feeling related to the contrastive values Security and Courage. If the person has access to only $40 \%$ of Courage, that is, a distance of $20 \%$ units from the ideal point, this will be related to a less positive feeling. If the distance to the ideal point is still larger in percent access (e.g., when the access of Courage is $20 \%$ or $100 \%$ such that in both cases the distance is $40 \%$ units), the related positive feeling will be still lower. The ideal points for negative feelings need not coincide with the ideal points for positive feelings and therefore it is of interest to measure negative and positive emotions separately. For instance, minimally negative feelings may be related to, for instance, $60 \%$ access to Security and $40 \%$ access to Courage. The example illustrates that for contrastive values the degree of positive or negative feelings 
has a maximum (positive feelings) or minimum (negative feelings) somewhere in between the end-points of the access continuum. For central values the ideal point for both positive and negative feelings is assumed to correspond to $100 \%$ access to the relevant value.

Since the ideal points for negative feelings need not coincide with the ideal points for positive feelings, the relative location of the ideal points may lead to decision conflicts between whether to choose an alternative that maximizes positive feelings (ideal point for positive feelings in focus) or to choose an alternative that minimized negative feelings (ideal point for negative feelings in focus). Of relevance to this is research on promotion and prevention focus in human motivation (Higgins 1997). Promotion focus corresponds to a motivation to move towards desired end states, which in terms of our theory would be a focus on the ideal point for positive feelings on the access continuum for a relevant pair of contrastive values. Prevention focus implies a motivation to move away from undesirable end states which in terms of our theory would mean a focus on the ideal point for negative feelings on the relevant value access continuum. Research has shown that people vary with respect to how much they are guided by one or the other of two types of foci in a given situation (Higgins et al. 1994), and, hence, may vary with respect to how they solve decision conflicts by favoring one or the other of differently located ideal points for positive or negative feelings.

We also propose that the relation between feelings and access to values is moderated by two factors. For contrastive values the location of the ideal point depends on the salience of the opposite value to a given contrastive value, or more exactly whether the opposite value to a given contrastive value is salient or not (i.e., activated). When the contrasting value (e. g. Courage) to a given value (e. g, Security) is salient, the ideal point will be located at a position between the two end-points of the access continuum (e.g., at $40 \%$ Security and $60 \%$ Courage between the end-points $0 \%$ Security $/ 100 \%$ Courage and 100\% Security/0\% Courage). If a contrastive value's opposite is not salient, decreasing the access to it means absence of access to the value and no increase of the opposite value. The ideal point is then located at maximal access to the value. For central values, the degree of positive feeling increases monotonically with increased access and the degree of negative feeling increases monotonically with decreased access.

The other moderating factor is the centrality of the values. If we interpret central and contrastive values as being on different levels in a value hierarchy where higher-level values (more central in the value structure) aggregate feelings associated with lower-level values (less central in the value structure), it follows that access to central values will lead to a greater range of positive and negative feelings than contrastive values. That is, maximal access to central values is expected to lead to more positive feelings and less negative feelings than contrastive values. Correspondingly, minimal access to central values are expected to lead to less positive and more negative feelings than for contrastive values.

\section{Study 2}

To empirically test the theoretical propositions above, we collected ratings of the strength of positive and negative feelings for different levels of access to central or contrastive values. For the contrastive values, the opposite was either salient or non-salient for different groups of participants. The following hypotheses were tested.

Hypothesis 1 states that $F_{p}$ and $F_{n}$ (i. e., ratings of positive and negative feelings, respectively) will be linearly related to value access for both central values and contrastive values with non-salient opposite with positive slopes for positive feelings and negative slopes for negative feelings. The ideal point in these cases equals 1 implying that Eq. 1 reduces to $F_{p}=a_{p}+b_{p} A$ for positive feelings and $F_{n}=a_{n}+b_{n}(1-A)$ for negative feelings.

Hypothesis 2 states that for contrastive values with a salient opposite, positive feeling ratings will be highest and negative feeling ratings will be lowest at an access level between $\mathrm{A}=0$ (minimal access) and $\mathrm{A}=1$ (maximal access). The ideal point has this intermediate position for contrastive values when the opposite value is salient.

Hypothesis 3 states that the ideal points for positive and negative feelings (operationalized as the access level associated with the highest and lowest feeling rating for positive and negative feelings, respectively) are expected to have different locations for at least some pairs of contrastive values.

Hypothesis 4 addresses the assumption that central and contrastive values are on different levels in a value hierarchy where higher level values (more central in the value structure) aggregate feelings associated with lower level values (less central in the value structure). From this we expect that feelings cover a greater range from weak to strong for central values than for contrastive values with non-salient opposite. The weakest and strongest feelings are thus weaker and stronger, respectively, for central than contrastive values.

Hypothesis 5 states that Eq. 1 describes the relation between feeling ratings and access level, if the access level associated with the highest and lowest mean rating, for positive and negative feelings, respectively, is inserted as an estimate of $I$ in the equation. There will thus be a linear relation between $F_{p}$ and $\left(1-\left|A-I_{p}\right|\right)$ and between $F_{n}$ and $\left|A-I_{n}\right|$, 
independently of whether $I$ is assumed to be 1 (central values, contrastive values with opposite value non-salient) or whether it is estimated from empirical data.

Hypothesis 6 complements Hypothesis 4 by using Eq. 1 for testing that higher level central values, as a result of aggregating feelings from lower level values, lead to a greater range of ratings than for comparable contrastive values, that is, contrastive values with non-salient opposite. If we assume that feelings are linearly related to value access for central values and contrastive values (Hypothesis 1) then it will be true that $F_{\text {min }}$ (minimal feeling rating) can be predicted from $\mathrm{F}_{\min }=\mathrm{a}$ and that $F_{\max }$ (maximal feeling rating) can be predicted from $\mathrm{F}_{\max }=\mathrm{a}+\mathrm{b}$, where $a$ is the additive constant and $b$ is the multiplicative constant in Eq. 1. This means that as a result of the greater range of ratings assumed for central values (lower $F_{\text {min }}$ and higher $F_{\text {max }}$ ) as compared to contrastive values (Hypothesis 4), $a$ will be lower and $\mathbf{a}+\mathrm{b}$ will be higher for central values than for comparable contrastive values. This means in turn that also $b$ will be greater for central values than for comparable contrastive values, since the lower value of $a$ in $\mathrm{a}+\mathrm{b}$ for central values, combined with the prediction that $\mathrm{a}+\mathrm{b}$ is greater, implies that $b$ must be greater for central values than for comparable contrastive values. Thus, Hypothesis 6 states that the additive constant $a$ is smaller, and the multiplicative constant $b$ is greater in Eqs. $1 \mathrm{a}$ and $1 \mathrm{~b}$ for central values than for contrastive values with non-salient opposite.

Hypothesis 7 addresses the question whether the additive constant $a$ and multiplicative constant $b$ of the relation between feeling strength and distance to the ideal point (Hypothesis 5) is independent of whether it corresponds to maximal value access or to an intermediate position of the ideal point (Hypothesis 2). In line with the parsimonious assumption that there is a common basis for how feelings are related to value access in the two cases, we hypothesize that $a$ and $b$ do not differ between maximal or intermediate positions of the ideal point.

\section{Method}

Fifty-four undergraduates at University of Gothenburg (Göteborg) were recruited to an online experiment. Ten were men, and 44 were women. Their mean age was 28.90 years and varied between 20 and 88 years $(S D=11.41)$. The participants received a lottery ticket worth the equivalent of US\$ 4 in compensation. Sample size was determined based on pilot studies.

The instructions read as follows (translated from Swedish): "This is a survey of values that may be important in life and feelings that are related to them. First, enter your gender and age. On each screen, one or two values are then presented. You should assess how much negative and positive feeling you experience when you have access to $0 \%$, $25 \%, 50 \%, 75 \%$ or $100 \%$ of these values. Examples of positive feelings include satisfied, happy, or cheerful. Examples of negative feelings include dissatisfied, sad, or dismissed." The values corresponded to the contrastive values presented in Table 1, and the central values presented in Table 2 . The ratings were made on two separate scales, one ranging from "no negative feeling" (0) to "strong negative feeling" (8) and the other ranging from "no positive feeling" (0) to "strong positive feeling" (8) The orders between the values were randomized individually.

The participants were randomly assigned to two approximately equally large groups. The first group received no information, implying minimal salience of the opposites of the contrastive values. For example, for the value Security and with $25 \%$ access to this value, the instructions read before presenting the scales for the ratings of positive and negative feelings: "Mark on the scales below how you feel when having access to $25 \%$ access to security". Both the central and contrastive values were presented. The second group received the same information but the opposites were specified, implying full salience of the opposite (see Table 1 for the definitions of opposites). For example, in the same example the instructions read: "Mark on the scales below how you feel when having $25 \%$ access to security and $75 \%$ access to courage". Only the contrastive values were presented.

\section{Results and discussion}

Figures 3 and 4 show mean ratings of positive and negative feelings as a function of value access to central values and to the contrastive values in the two salience conditions. In line with Hypothesis 1, the functions are approximately linear for central values as well as for contrastive values when the opposite contrastive value was non-salient (i.e., not presented).

In line with Hypothesis 2, for contrastive values with salient opposites the functions peak in the middle of the access continuum, with the peak corresponding to the access level associated with the highest mean rating for positive feelings and the access level with the lowest mean rating for negative feelings. The mean ratings are in this case plotted for both possible access levels for a given value since the left-right location of the two values in the value pair can be seen as arbitrary.Table 3 presents means and standard deviations of the estimated ideal points for each contrastive value pair. Ideal points are estimated as the access level associated with the highest rating of positive feelings and the access level associated with the lowest rating of negative feelings for each value pair and participant. If several access levels have maximum positive feelings or minimum negative feelings, 


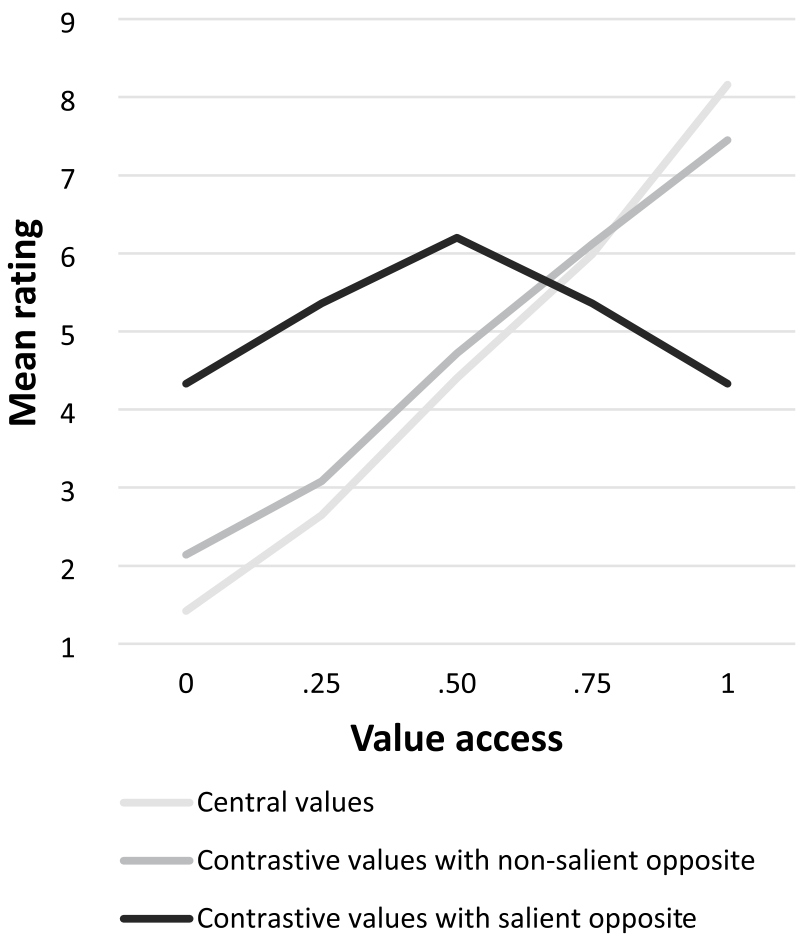

Fig. 3 Mean ratings of positive feelings as a function of access to central values and contrastive values with non-salient or salient opposite

the means of these access levels were used. In line with Hypothesis 2, the estimated ideal points are located in the middle part of the access continuum, although in most cases closer towards the value associated with one end-point. The standard deviations are approximately 0.30 indicating that the ideal-points vary across participants over the 0 to 1 range of the access continuum. In line with Hypothesis 3, the locations of the ideal points for positive and negative emotions differ significantly (dependent t-tests, $p<.05$ ) for three of the four value pairs. This is particularly evident for the value pair Security-Courage. Here the results indicate that in order for the participants to feel maximally positive, they should strive for more security than courage ( $I_{p}$ closer to security than courage), at the same time as they should strive for more courage than security to feel minimally negative $\left(I_{n}\right.$ closer to courage than security) (see Table 3 ).

To test whether the range from lowest to highest mean rating is dependent on whether the values are central or contrastive with non-salient opposites, $t$-tests were computed between the mean ratings for the two types of values for minimum access level $(\mathrm{A}=0)$ and maximum access level $(A=1)$, respectively. The relevant means are presented in Table 4 and can also be inferred from the end-points of the linear functions in Figures 3 and 4. In line with Hypothesis 4 , all differences between relevant means were statistically significant in the expected directions. Thus, $F$ for positive

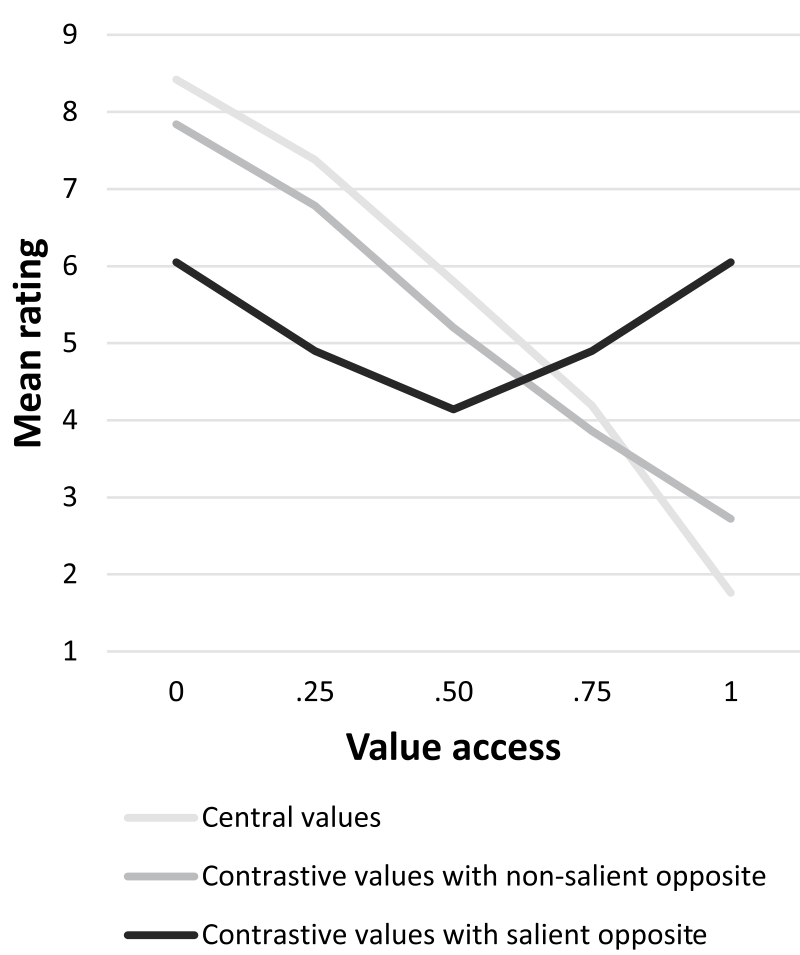

Fig. 4 Mean ratings of negative feelings as a function of access to central values and contrastive values with non-salient or salient opposite

feelings when $\mathrm{A}=0$ is lower for central values than for contrastive values and higher for negative feelings. When $\mathrm{A}=$ 1 , the reverse pattern is found, that is $F$ for positive feelings is higher for central values than contrastive values and lower for negative feelings.

Since Hypothesis 4 was confirmed, and given that Eqs. 1a and $1 \mathrm{~b}$ show good fits to the data (Hypothesis 5), $a$ and $b$ are expected to depend on whether the values are central or contrastive (Hypothesis 6), but independent of the position of $I$ (Hypothesis 7), that is independent of whether an opposite contrastive value is non-salient or salient. The multiplicative parameter $b$ and the additive parameter $a$ in Eqs. $1 \mathrm{a}$ and $1 \mathrm{~b}$, as well as the fits of the equations, were estimated from separate regression analyses of the positive and negative feelings ratings of central and contrastive values, respectively.

The results of the regression analyses are shown in Table 5. The fits of the equations vary from $R^{2}=.41$ (negative feelings, contrastive values with salient opposite) to $\mathrm{R}^{2}=0.75$ (positive feelings, central values). On the whole, and in line with Hypothesis 5, the fits are good (cf. Cohen 1988), although the fit is lower for contrastive values with salient opposites than with non-salient opposites. The lower fit could be expected to result from the restricted range of the ratings in this case (cf. the end-points of the curves in Figs. 3, 4). In line with Hypothesis 6, the additive constant $a$ for positive feelings is lower for central values than for 


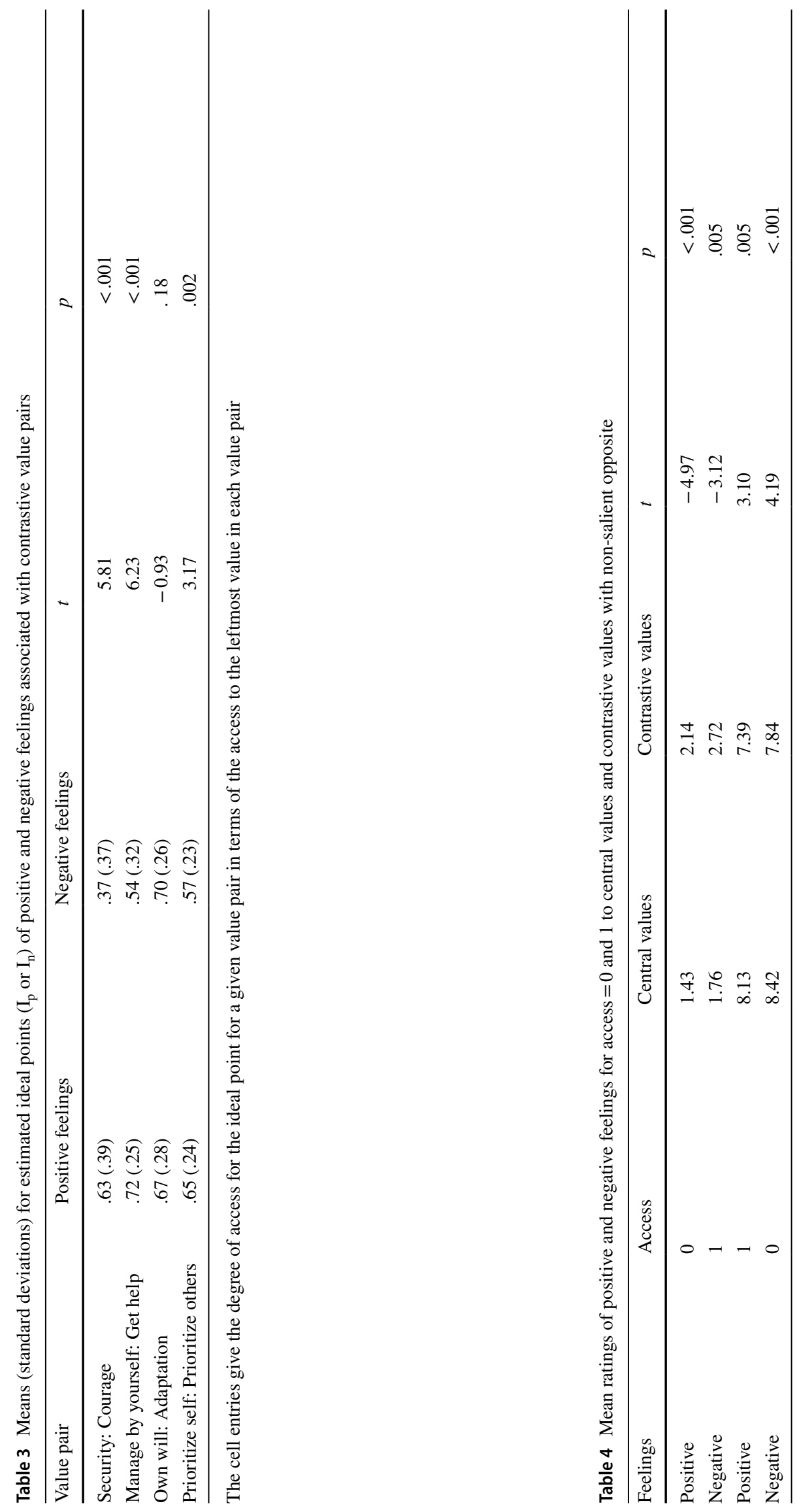


Table 5 Estimates of coefficients associated with a and $\mathrm{b}$ from regression analyses of ratings of positive feelings as a function of $\left(1-\mathrm{I}_{\mathrm{p}}-\mathrm{A}\right)$ and of ratings of negative feelings as a function of $I_{n}-A \mid$ for different value types

\begin{tabular}{llllllll}
\hline Value type & \multicolumn{2}{l}{ Positive feelings } & & \multicolumn{3}{l}{ Negative feelings } \\
\cline { 2 - 3 } \cline { 6 - 7 } & $\mathrm{a}$ & $\mathrm{b}$ & $\mathrm{R}$ & & $\mathrm{a}$ & $\mathrm{b}$ & $\mathrm{R}^{2}$ \\
\hline Central values & $.02(.01)$ & $.85(.02)$ & .75 & $.16(.01)$ & $.81(.02)$ & .68 \\
$\begin{array}{l}\text { Contrastive value } \\
\text { with non-salient } \\
\text { opposite }\end{array}$ & $.12(.01)$ & $.70(.02)$ & .61 & $.18(.01)$ & $.69(.02)$ & .57 \\
$\begin{array}{l}\text { Contrastive with sali- } \\
\text { ent opposite }\end{array}$ & $.14(.01)$ & $.68(.01)$ & .47 & $.26(.01)$ & $.68(.02)$ & .41 \\
\hline
\end{tabular}

Standard errors are given within parentheses contrastive values with non-salient opposites and the multiplicative constant $b$ is larger in the same comparison for both positive and negative feelings. For negative feelings $a$ is about the same for central values and contrastive values with non-salient opposites. In line with Hypothesis 7, for positive as well as negative feelings, $a$ and $b$ are close to each other for contrastive values across non-salient opposites and salient opposites. But $a$ differs for negative feelings across the two conditions. Thus, it seems that with the possible exception for $a$ for negative feelings, in line with Hypothesis 7 we cannot reject the statistical hypothesis that the parameters of Eqs. $1 \mathrm{a}$ and $1 \mathrm{~b}$ are independent of whether the ideal point is located at a maximal or at an intermediate access level.

In order to determine the statistical significance of the patterns observed for the parameters $\mathrm{a}$ and $\mathrm{b}$ across positive and negative feelings, mixed model analyses were carried out, where ratings of positive and negative feelings, respectively, are compared for central values and contrastive values with non-salient opposites (within-group comparison) and for contrastive values with non-salient and salient opposites, respectively (between-group comparison). The alpha level was set to 0.05 . The fixed factor in each analysis was centrality (i.e., central values versus contrastive values with nonsalient opposite) and salience (i.e., contrastive values with non-salient opposite versus contrastive values with salient opposite), calculations of (1-|A-II) and IA - Il for positive and negative feelings are entered as covariates. Repeated measures are access level and value. A Toeplitz covariance structure is assumed, which implies that co-variances are assumed to be higher for access levels that are closer to each other. To check the validity of the chosen covariance structure, a base line test was made replacing the Toeplitz covariance structure with a compound symmetry structure (homogenous variances and co-variances). The fit of the model based on Toeplitz covariance structure was superior in all four tested cases with -2 Restricted Log Likelihood differences varying between 524 and 812 [corresponding to $\chi^{2}(80)$ and $\chi^{2}(40)$ for the within-group and between-group comparisons, respectively], $p<0.001$ in all cases.

The main effect in the analysis concerns whether including an additive constant (i.e., $a$ ) improves the fit significantly and the interaction of the fixed factor with the covariate concerns whether the fit is improved significantly by including a multiplicative constant (i.e., $b$ ). Table 6 presents estimates of main effects and of the interaction effects which correspond to differences in estimates of $a$ and $b$, respectively, between the cases that are compared in each analysis. It can be seen that in line with the observed pattern from the outcome of the regression analyses in Table 5, and also in line with Hypothesis 6, a was significantly lower for positive feelings for central vales as compared to contrastive values with non-salient opposites, and $b$ was significantly higher for both positive and negative feelings in this comparison. In the comparison of feelings associated with contrastive values with non-salient opposites and contrastive values with salient opposites, $a$ for negative feelings was significantly greater for contrastive values with non-salient opposites, which is not in line with Hypothesis 7, which states that $a$

Table 6 Estimates of fixed effects from mixed model analyses

\begin{tabular}{|c|c|c|c|c|c|c|c|}
\hline \multirow[t]{2}{*}{ Tested effect } & \multirow[t]{2}{*}{ Parameter } & \multicolumn{3}{|c|}{ Positive feelings } & \multicolumn{3}{|c|}{ Negative feelings } \\
\hline & & Est & $t$ & $p$ & Est & $t$ & $p$ \\
\hline \multirow[t]{2}{*}{ Central values vs. contrastive values with non-salient opposite } & a & -.09 & 4.68 & .027 & -.05 & 2.79 & .006 \\
\hline & $\mathrm{b}$ & .16 & -5.07 & $<.001$ & .14 & -4.47 & $<.001$ \\
\hline \multirow{2}{*}{$\begin{array}{l}\text { Contrastive values with non-salient opposite vs. contrastive } \\
\text { values with salient opposite }\end{array}$} & a & -.02 & -0.70 & $<.001$ & .08 & 2.16 & .03 \\
\hline & $\mathrm{b}$ & -.03 & -.67 & $<.001$ & .06 & 1.23 & .22 \\
\hline
\end{tabular}


and $b$ are independent of salience of opposite contrastive value. Yet, in line with Hypothesis 7 no significant effects were observed for the three remaining differences for this comparison.

It can be concluded that our hypotheses of how feeling ratings as a function of value access are related to value centrality (central versus contrastive values) and to salience (salience versus non-salience of opposite contrastive value) were largely confirmed in regression analyses and the subsequent mixed model analyses.

\section{General discussion}

In this paper we presented a theory that integrates values and emotions that both are factors motivating human behavior. We found empirical evidence for that positive and negative feelings are linked to our new proposed value structure. The value structure is related to Schwartz et al.'s (2012) value structure, but there are some important differences. First, we have investigated the structure of values by means of ratings of how compatible one value is with another value, and hence, focused on how individuals cognitively represent values, whereas Schwartz and other researchers have examined value structures based on correlations across individuals. In a previous study based on correlations across individuals (Montgomery et al. 2013, Experiment 1), the same structure for contrastive values was found as revealed by compatibility ratings in the present study. Still, it remains to be investigated how central values are located in a value structure based on individual differences. Note, however, that only the present approach to measuring value structure is adequate when relationships between values and emotions are examined since our theory concerns intra-individual relations between values and emotions. Second, and related to the previous point, we have found evidence for describing values not only in terms of their positions along the periphery of the circumplex, as has been the case in Schwartz' research, and also is true for the contrastive values in the present set of values. In addition, we have identified a set of values - central values - that ate located inside the periphery and which we interpret as higher-order values that may be accessed through access to lower level contrastive values. Third, we have found evidence for systematic relationships between values and positive and negative feelings. Our findings in this respect will be discussed next.

We noted in the introduction that no systematic studies of the relationship between values and emotions seem to have been conducted on the intra-individual level. In Study 2 our aim was to find a general pattern within individuals of how positively and negatively valenced feelings are linearly related to the access and salience of different values, disregarding specific other qualities of positive or negative feelings. We thus showed that Eq. 1 predicted both rated positive and negative feelings for access to contrastive values when the opposite value was non-salient or salient as well as for central values. We also found that the additive parameter $a$ and the multiplicative parameter $b$ in Eq. 1, which both are linked to value centrality (with central values being more central and contrastive values being less central in the value structure) made a significant contribution to the fit of Eq. 1 to the empirical data. The fitted numerical values of the parameters for contrastive and central were in line with the assumption that central values (representing a higher level in the value hierarchy) aggregate feelings associated with contrastive lower-order values. This means that there are two independent empirical bases for the distinction between these two types of values, that is the ratings of compatibility between values in Study 1, and the feeling ratings connected to values in Study 2.

We suggest that the interplay between activation of contrastive and central values and feelings linked to specific values is an important topic for future research of how values influence decision making and ultimately human behavior. Another avenue for future research is to examine the feelings that are evoked when the individual is confronted with realistic (and not only imagined) possibilities of obtaining or losing access to different values. Our study is limited to measuring expected or anticipated conscious elements of emotions (referred to as "core affects" by Russell 2003). We recognize that anticipated feelings are cognitive representations but it has still been shown that they vary in the same positive-negative valence dimensions as actual feelings do (Västfäll et al. 2004; Västfjäll and Slovic 2013). Future research is needed to investigate whether activation of values evokes the actual feelings as one may then expect. Experimental studies should be conducted in which people are faced with different combinations of access to contrastive values (e.g., in terms of number of time units to volunteer for a task giving opportunities for showing courage), which would evoke excitement as well as fear, as opposed to number of time units of being in a secure situation (which would evoke calmness and boredom). At the same time, it is important to note that the present study reveals how people think about how feelings are evoked by activated values, which would be relevant for how people choose to approach or avoid situations involving activation of different values.

The present study has implications for how people may manage to resolve decision conflicts. Awareness of these implications could be useful in developing decision aids. Often good decisions could not be made by only attending central values, since they do not not motivate a clear direction of action. To resolve a decision conflict, the decision maker needs to be aware of conflicts between opposing contrastive values that are involved in the conflict and, as a consequence, search for an optimal balance between the 
contrastive values in combination with, as will be discussed below, a goal to maximize the expected access to central values. The resolution of a decision conflict could be further complicated by approach-avoidance conflicts resulting from different locations of the ideal points for positive and negative feelings, respectively. When an opposite contrastive value is salient, or could be made salient, the balance between value access, for both positive and negative feelings, rather than the maximization of access to single values should be expected to be a fundamental goal of decision making. This means in turn that in these cases it would be misleading to define contrastive values as desirable goals. Rather, desirable goals should be defined as an optimal balance. Note that this view is consistent with multiple-criterion analysis of decision making (Köksalan et al. 2011), although this analysis does not explicitly define conflicting goals in terms of contrastive values and expected feelings evoked by activation of specific values.

The alternatives in decision making may be more or less close to the ideal points located in between the maximal access to opposing opposite values. In line with previous theorizing, we propose that the extent to which an alternative is favoured is related to how close it is to an ideal point (Restle 1961; Zeleny 1976; Zakay and Barak 1984), which in our case corresponds to a combination of levels of access to opposing contrastive values. Which role does then the expected access to central values play? We propose that the decision maker locates the ideal point such that it gives the best perceived possibility to maximize the access to those central values that are most important for the decision maker in terms of expected feelings associated with the decision. The situational salience or access to certain central values can be assumed to influence the location of the ideal point. Research is needed to examine the validity of this account of decision making in terms of contrastive and central values, in conjunction with feelings related to these values.

The extent to which people find a balance between contrastive values may vary across individuals. Some persons may feel only bored and never relaxed when they are secure and feel excited but not afraid when being courageous. Others may to an equal degree have positive and negative feelings when confronted with contrastive values, such as Security and Courage. A negative feeling signals that one has too much access to a contrastive value and too little to its opposite, and if a positive feeling signals that normal access to a contrastive value is reached, this is a sign of a more balanced personality, which presumably as a rule is better adapted to multi-facet environments. This idea may be used to develop a test of how balanced value structures people have to indicate if they are well adjusted.

On a cultural level, a balanced relationship between opposing value orientation is stressed in the Chinese Yin and Yan philosophy (Fang 2018). In Western cultures, on the other hand, the imbalance is presumably more often between individualistic values (more emphasized) and collectivistic values (less emphasized) (Johanloo and Jarden 2016; Schwartz 2012; Triandis et al. 1993). In line with this hypothesis, Johanloo and Jarden (2016) recently found that Hedonism (focus on private enjoyment) is more strongly related to Happiness (positive feeling) in more individualistic than in collectivistic cultures. We feel that an interesting task for future research is to study cultural differences in the importance and conception of balanced value relationships, and how such differences are related to positive and negative feelings.

In conclusion, the present study has led to insights about the structure of human values and how it is linked to positive and negative feelings depending on the extent to which and how contrastive and central values are activated. Our findings invite future research on topics such as the malleability of the conceived relationship between contrastive values and additional knowledge on the interplay between people's feelings, emotions and values, particularly for decisions preceding behavioral outcomes. This has the promise of developing a theory that not only integrates values and emotions but also specify how they interact in determining human behaviour.

Acknowledgements Open access funding provided by University of Gävle. This research was financially supported by Grant 2006-721540797-22 from the Swedish Research Council to Henry Montgomery

Open Access This article is licensed under a Creative Commons Attribution 4.0 International License, which permits use, sharing, adaptation, distribution and reproduction in any medium or format, as long as you give appropriate credit to the original author(s) and the source, provide a link to the Creative Commons licence, and indicate if changes were made. The images or other third party material in this article are included in the article's Creative Commons licence, unless indicated otherwise in a credit line to the material. If material is not included in the article's Creative Commons licence and your intended use is not permitted by statutory regulation or exceeds the permitted use, you will need to obtain permission directly from the copyright holder. To view a copy of this licence, visit http://creativecommons.org/licenses/by/4.0/.

\section{References}

Baumeister, R. F. (2007). How emotion shapes behaviour: Feedback, anticipation, and reflection, rather than direct causation. Personality and Social Psychology Review, 167, 167-203. https://doi. org/10.1177/1088868307301033.

Beck, A. T., Rush, A. J., Shaw, B. F., \& Emery, G. (1979). Cognitive depression theory. New York: Guildford.

Cohen, J. (1988). Statistical power analysis for the behavioural sciences (2nd ed.). Hillsdale, NJ: Lawrence Erlbaum Associates.

Coombs, C. H. (1950). Psychological scaling without a unit of measurement. Psychological Review, 5, 145-158.

Fang, T. (2018). Yin Yang: A new perspective on culture. Management and Organizational Review, 8(1), 25-50. https://doi.org/10.111 1/j.1740-8784.2011.00221.x. 
Feldman Barrett, L., Lewis, M., \& Haviland-Jones, J. M. (Eds.). (2016). Handbook of emotions (4th ed.). New York: Guilford Press.

Higgins, E. (1997). Beyond pleasure and pain. American Psychologist, 52(12), 1280-1300. https://doi. org/10.1037/0003-066X.52.12.1280.

Higgins, E. T., Roney, C. J., Crowe, E., \& Hymes, C. (1994). Ideal versus ought predilections for approach and avoidance distinct selfregulatory systems. Journal of Personality and Social Psychology, 66, 276-286. https://doi.org/10.1037/0022-3514.66.2.276.

Johanloo, M., \& Jarden, A. (2016). Individualism as the moderator of the relationship between hedonism and happiness: A study in 19 nation. Personality and Individual Differences, 94, 149-152. https ://doi.org/10.1016/j.paid.2016.01.025.

Johnson-Laird, P. N., \& Oatley, K. (1992). Basic emotions, rationality, and folk theory. Cognition and Emotion, 6, 201-223. https://doi. org/10.1080/02699939208411069.

Köksalan, M., Wallenius, J., \& Zionts, S. (2011). Multiple criteria decision making: From early history to the 21 st century. Singapore: World Scientific.

Koopman-Holm, B., \& Matsumoto, D. (2011). Values and display rules for specific emotions. Journal of Cross-Cultural Psychology, 42, 355-371. https://doi.org/10.1177/0022110362753.

Leszkowicz, E., Linden, D. E. J., Maio, G. R., \& Ihssen, N. (2017). Neural evidence of motivational conflict between social values. Social Neuroscience, 12(5), 494-505. https://doi. org/10.1080/17470919.2016.1183517.

Maio, G. R. (2016). The psychology of human values. Abingdon: Routledge.

Maio, G. R., Pakizeh, A., Cheung, W.-Y., \& Rees, K. J. (2009). Changing, priming and acting on values: Effects via motivational relations in a circular model. Journal of Personality and Social Psychology, 97, 699-715. https://doi.org/10.1037/a0016420

Mesquita, B., \& Frijda, N. H. (2011). Regulation. Cognition and Emotion, 25, 782-784. https://doi.org/10.1080/02699931.2011.58682 4.

Montgomery, W., Montgomery, H., \& Gärling, T. (2013). Reconceptualizing value structure. Working paper, Department of Psychology, Gothenburg University. Retrieved from https://www.resea rchgate.net/publication/310600196_Reconceptualizing_value _structure.

Moors, A., Ellsworth, P. H., Scherer, K. R., \& Frijda, N. H. (2013). Appraisal theories of emotion; State of the art and future development. Emotion Review, 5, 119-124. https://doi.org/10.1177/17540 73912468165

Nelissen, R. M. A., Dijker, A. J. M., \& de Vries, N. K. (2007). Emotions and goals: Assessing relations between values and emotions. Cognition and Emotion, 21, 902-911. https://doi.org/10.1080/02699 930600861330

Restle, F. (1961). Psychology of judgment and choice: A theoretical essay. Oxford: Wiley.

Russell, J. A. (1980). A circumplex model of affect. Journal of Personality and Social Psychology, 39(6), 1161-1178. https://doi. org/10.1037/h0077714.

Russell, J. A. (2003). Core affect and the psychological construction of emotion. Psychological Review, 110, 145-172. https://doi. org/10.1037/0033-295X.110.1.145.

Russell, J. A., \& Feldman Barrett, L. (1999). Core affect, prototypical emotional episodes, and other things called emotion. Journal of Personality and Social Psychology, 76, 805-819.

Scherer, K. R. (1999). Appraisal theory. In T. Dalgleish \& M. Power (Eds.), Handbook of cognition and emotion (pp. 637-663). Chichester: Wiley.

Schwartz, S. H. (1992). Universals in the content and structure of values: Theoretical advances and empirical tests in 20 countries. In
M. P. Zanna (Ed.), Advances in experimental social psychology (Vol. 24, pp. 1-65). San Diego, CA: Academic.

Schwartz, S. H. (2007). Universalism values and the inclusiveness of our moral universe. Journal of Cross-Cultural Psychology, 38, 711-728. https://doi.org/10.1177/00220107308992.

Schwartz, S. H. (2010). Basic values: How they motivate and inhibit prosocial behaviour. In M. Mikulincer \& P. Shaver (Eds.), Prosocial motives, emotions, and behaviour: The better angle of our nature (pp. 221-241). Washington, DC: American Psychological Association.

Schwartz, S. H. (2012). An overview of the Schwartz theory of basic values. Online Readings in Psychology and Culture. https://doi. org/10.9707/2307-0919.1116.

Schwartz, S. H., \& Bardi, A. (2001). Value hierarchies across cultures, taking a similarities perspective. Journal of Cross-Cultural Psychology, 32, 268-290. https://doi.org/10.1177/002202210103200 3002 .

Schwartz, S. H., \& Boehnke, K. (1992). Evaluating the structure of human values with confirmatory factor analysis. Journal of Research in Personality, 38, 230-255. https://doi.org/10.1016/ S0092-6566(03)00069-2.

Schwartz, S. H., Cieciuch, J., Vecchione, M., Davidov, E., Fischer, R., Beierlein, C., et al. (2012). Refining the theory of basic human values. Journal of Personality and Social Psychology, 103, 663688. https://doi.org/10.1037/a029393.

Silfver, M., Helkama, K., Lönnqvist, J.-E., \& Verkasalo, M. (2008). The relation between value priorities and proneness to guilt, shame, and empathy. Motivation and Emotion, 32(2), 69-80. https ://doi.org/10.1007/s11031-008-9084-2.

Tamir, M., Schwartz, S. H., Cieciuch, J., Riediger, M., Torres, C., Scollon, C., et al. (2016). Desired emotions across cultures: A value based account. Journal of Personality and Social Psychology, 111, 67-82. https://doi.org/10.1037/pssp0000072....

Triandis, H. C., McCusker, C., Betancourt, H., Iwao, S., Leung, K., et al. (1993). An etic-emic analysis of individualism and collectivism. Journal of Cross-Cultural Psychology, 24, 366-383. https:// doi.org/10.1177/0022022193243006.

Västfjäll, D., Gärling, T., \& Kleiner, M. (2004). Preference for mood, emotional reaction, and anticipated emotional reaction. Scandinavian Journal of Psychology, 45, 25-34. https://doi.org/10.111 1/j.1467-9450.2004.00375.x.

Västfjäll, D., \& Slovic, P. (2013). Cognition and emotion in judgment and decision making. In M. D. Robinson, E. R. Watkins, \& E. Harmon-Jones (Eds.), Handbook of cognition and emotion (pp. 252-271). New York: Guilford Press.

Verplanken, B., \& Holland, R. (2002). Motivated decision making: Effects of activation and self-centrality of values on choices and behaviour. Journal of Personality and Social Psychology, 82, 434-447. https://doi.org/10.1037/0022-3514.82.3.434.

Yik, M., Russell, J. A., \& Steiger, J. H. (2011). A 12-point circumplex structure of core affect. Emotion, 11, 705-731. https://doi. org/10.1037/a0023980.

Zacharopoulos, G., Hanel, P. H. P., Lancaster, T. M., Ihssen, N., Drakesmith, M., Foley, S., et al. (2017). Nonlinear associations between human values and neuroanatomy. Social Neuroscience, 12, 673-684. https://doi.org/10.1080/17470919.2016.1229215....

Zakay, D., \& Barak, A. (1984). Meaning and career-decision making. Journal of Vocational Behavior, 24, 1-14.

Zeleny, M. (1976). The attribute-dynamic attitude model. Management Science, 23, 12-25.

Publisher's Note Springer Nature remains neutral with regard to jurisdictional claims in published maps and institutional affiliations. 